\title{
Manipulating the thermal and dynamic mechanical properties of polydicyclopentadiene via tuning the stiffness of the incorporated monomers
}

https://doi.org/10.1515/epoly-2019-0037

Received December 10, 2018; accepted March 02, 2019.

\begin{abstract}
The application of polydicyclopentadiene (polyDCPD) as a high-performance thermosetting resin is often hindered by the simplicity and limitation of the polymer structure, making it unlikely to improve their thermal and dynamic mechanical properties by further optimizing the polymerization conditions. In this study, we developed a copolymer system which consisted of dicyclopentadienes and various designed monomers as excellent curing agents. The incorporated monomers bearing different stiffness and rigidity contain two active functional groups at the end of the structures and are capable of reinforcing original polyDCPD. The incorporated monomers notably enhanced the thermal and dynamic mechanical properties of polyDCPD. Besides that, the relationship between the stiffness of the monomer and the thermal and dynamic mechanical properties of polyDCPD was evaluated in detailed. Because of the simplicity and adjustability of copolymerization approach, optimal conditions of the copolymers with best property-reinforcing capability were systemically identified. The optimal materials displayed desired thermal and dynamic mechanical property and markedly outperformed the original polyDCPD.
\end{abstract}

Keywords: polyDCPD; stiffness of the monomer; reinforcing; thermal and dynamic mechanical properties

\footnotetext{
* Corresponding author: Yubin Zheng, State Key Laboratory of Fine Chemicals, Province Key Laboratory of Polymer Science and Engineering, Department of Polymer Science and Engineering, Dalian University of Technology, Dalian, China, 116024, e-mail: zybwl@163.com.

Jia Kuang, Nan Zheng and Chenglin Liu, State Key Laboratory of Fine Chemicals, Province Key Laboratory of Polymer Science and Engineering, Department of Polymer Science and Engineering, Dalian University of Technology, Dalian, China, 116024
}

\section{Introduction}

Polydicyclopentadiene (polyDCPD) prepared by the ringopening metathesis polymerization (ROMP) method (1-3) is a kind of highly crosslinked and thermosetting material. During the past decades, it has attracted extensive interests in both academic and industrial fields for the widespread applications in latexes, low-density materials and selfhealing materials (4-7). It has been widely and broadly utilized to make the bumpers, body panels and other components for trucks, buses, tractors, water vehicles, and heavy-duty construction equipment due to the advantages of low cost, low density with high toughness, high impact strength and corrosion resistance $(8,9)$. However, despite its extensive use in both academic and industrial fields, polyDCPD poses a major drawback which extensively hampers their further applications, that is, the limited thermal stability and dynamic mechanical property issues due to the uncontrolled and fast polymerization rate, as well as the high density of the unsaturated bonds existing in the polymer structures (10-12). The thermal and dynamic mechanical properties of the polyDCPDbased materials have been notably improved by preparing various composites with reinforcing materials. Since the chemical nature of the incorporated materials determined their property behavior, it is significant and useful to evaluate the relationship between the chemical structures and the properties, which could provide a rational design to manipulate the properties. It has been studied in our previous work that by optimizing the polymerization conditions including changing the catalyst structures, loadings, the polymerization temperature and reaction time, unfortunately the thermal property and dynamic mechanical property cannot be furthermore improved (13). Recently, an effective strategy to reinforce the thermal and dynamic mechanical property of polyDCPD is to incorporate other functional monomers into the polyDCPD, which could decrease the polymerization rate and improve the crosslinking (11,14-17). It has been 
well known and reported that incorporation of the rigid structures could promote the thermal stability of polyDCPD by forming the stiff and crosslinked copolymers (18-22). Recently, the aromatic domains such as the styrene have been introduced into the polyDCPD as well as other thermosetting resins to enhance their thermal and mechanical properties (23). Among various rigid motifs, bismaleimide, as a category of high-performance thermosetting material, has attracted general interest (2327). For instance, the incorporation of phenolphthaleincontaining bismaleimide (PPBMI) indicated notably increased thermal stability and mechanical property (28). However, due to the super rigid structure of PPBMI and the compatibility with polyDCPD not as good as expected, severe phase separation could occur during the polymerization. In addition, the inhibition of the crosslinking as well as the generated obvious cracks would impede the improvement of the thermal and mechanical property (29).

As a result, the rigidity of the inserted monomer exhibited significant functionality in determining the thermal stability and mechanical property. Neither super rigid nor super flexible monomers would promote the improvement of the thermal and mechanical property. Till now, there is no related and systematical study on the relationship between the polyDCPD polymer and the rigidity of the inserted monomer to achieve the optimal copolymers with excellent thermal and mechanical properties.

Based on the above understandings on the reinforcing of polyDCPD, herein we incorporated various domains (OCPD, MPBM and OXDBMI) with different stiffness and rigidity into the design of original and unmodified polyDCPD, attempting to strengthen their thermal and mechanical properties and potentially manipulate the reinforcing abilities. The incorporated monomers are designed and classified based on their stiffness and rigidity. OCPD is a monomer with an aliphatic chain, which belongs to a flexible monomer in the modification library. MPBM is a bismaleimide-mimic structure with the rigid aromatic moiety, which belongs to the highly stiff monomer. OXDBMI is designed as a combination of both the flexible and the stiff properties into one monomer.

To this end, crosslinked copolymers bearing both polyDCPD and a library of incorporated modification monomers were synthesized and prepared. We hypothesized that the combination of both flexible and stiff properties into one monomer would allow optimal improvement in the thermal and mechanical properties via reinforcing the rigidity of the polymer as well as limiting the extent of incompatibility. To demonstrate such hypothesis, the effect of monomers bearing different structures on the thermal stability, dynamic mechanical property and the mechanical property were systemically explored.

\section{Experimental}

\subsection{Materials}

DCPD ( $>96 \%)$ was purchased from J\&K Chemical Co., Shanghai, China, and was used after treating with $\mathrm{CaH}_{2}$ followed by distillation under reduced pressure to remove any trace impurities. The first-generation Grubbs' catalyst [Ru] (Bis(tricycle hexylphosphine)benzylidine ruthenium (IV) dichloride) was prepared as previously reported $(13,28)$. The catalyst was lyophilized and stored at $4^{\circ} \mathrm{C}$ under a nitrogen atmosphere. Anhydrous tetrahydrofuran (THF), hexane, ether and other solvents were dried by a column packed with $4 \AA$ molecular sieves. 1, 1'-(Methylenedi-4,1-phenylene) bismaleimide (MPBM), 4-Nitrobenzhydrazide and 4-(4-Nitrophenoxy) benzoic acid were purchased from J\&K Chemical Co., Shanghai, China. All other chemicals were purchased from Tianjin Kermel Chemical Reagent Co., Ltd. (China) and used as received unless otherwise specified.

\subsection{Characterization}

Fourier transform infrared (FTIR) spectrum was obtained on a Nicolet 6700 FTIR spectrometer. Thermo gravimetric analysis (TGA) was performed on a PerkinElmer TGA-7 thermal analyzer. Samples of approximately $5 \mathrm{mg}$ were heated from room temperature to $800^{\circ} \mathrm{C}$ at a rate of $10^{\circ} \mathrm{C} \mathrm{min}^{-1}$ under a nitrogen atmosphere with a flow rate of $20 \mathrm{~mL} \mathrm{~min}^{-1}$. The dynamic mechanical analysis (DMA) of the polymers was performed on a TA Q800 instrument. Samples were analyzed in the three-point bending mode with amplitude of $20 \mu \mathrm{m}$ at a frequency of $1.0 \mathrm{~Hz}$ and were heated from room temperature to $220^{\circ} \mathrm{C}$ at a heating rate of $3^{\circ} \mathrm{C} \mathrm{min}{ }^{-1}$. The samples were prepared by compression product into rectangular bars as $40 \mathrm{~mm}$ in length, $7 \mathrm{~mm}$ in width, and $2 \mathrm{~mm}$ in thickness. The mechanical properties of the cured samples were evaluated from the flexural strength and flexural modulus according to the GB/T 9341-2008. Three-point bending tests were performed on a Universal Testing Machine (Instron 5567A) at a crosshead speed of $2 \mathrm{~mm} \mathrm{~min}^{-1}$. 


\subsection{Synthesis of polyDCPD}

PolyDCPD was prepared by the ring-opening metathesis polymerization (ROMP) method (4,5,13). DCPD was dissolved in the dry dichloromethane (DCM) solvent in an ice bath, followed by addition of the first-generation Grubbs' catalyst [Ru] (DCPD/[Ru] ratio: 1000/1). The polymerization system was transferred into a mould after the solvent was removed under vacuum and the resulting polymer was further cured at the following gradient temperature to complete the polymerization: $10^{\circ} \mathrm{C}$ for $1 \mathrm{~h}, 60^{\circ} \mathrm{C}$ for $2 \mathrm{~h}, 120^{\circ} \mathrm{C}$ for $2 \mathrm{~h}$ and then at $150^{\circ} \mathrm{C}$ for $2 \mathrm{~h}$ (Scheme 1).

\subsection{Synthesis of 5-octylcyclopenta-1,3-diene (OCPD)}

The cyclopentadienyl sodium (CPD-Na) was synthesized via the reaction between cyclopentadiene and sodium hydride according to the previously reported method (30). Then 1-chlorooctane was added into the CPD-Na dissolving in THF and the reaction was kept stirring until the CPD-Na was totally reacted. The obtained OCPD was purified by silica column (Scheme 2).

\subsection{Synthesis of 1-[4-[5-[4-[4-(2,5-Dihydro- 2,5-dioxo-1H-pyrrol-1-yl) phenoxy] phenyl]-1,3,4-oxadiazol-2-yl] phenyl]- 1H-pyrrole-2,5-dione (OXDBMI)}

4-Nitrobenhydrazide, 4-(4-nitrophenoxy) benzoic acid and polyphosphoric acid were mixed and stirred at $120^{\circ} \mathrm{C}$ for $6 \mathrm{~h}$, followed by pouring into distilled water after cooling down and collecting the solid precipitate. The solid precipitate was then mixed with activated charcoal, $\mathrm{FeCl}_{3}$ and 2-methoxyethanol and heated to $110^{\circ} \mathrm{C}$, followed by dropwise addition of $85 \%$ hydrazine monohydrate. The reaction was kept at $110^{\circ} \mathrm{C}$ for another $10 \mathrm{~h}$ under a nitrogen atmosphere and then separated by filtration. The obtained product was then dissolved with acetone and followed by dropwise addition of maleic anhydride. The reaction was stirred for $9 \mathrm{~h}$ after the solution turned into clear. The

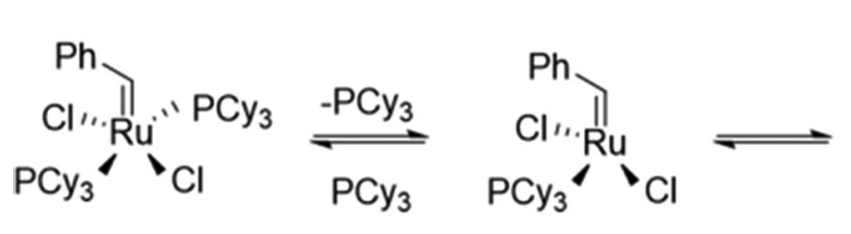<smiles>C=CCC1CC(C=CC2CC(C=CC3CC(C=CC4CC(C=C)C5C=CCC45)C(C=CC4CC(C=CC5CC(C=C)C6C=CCC56)C5C=CCC45)C3CC=CC3CC(C=C)C4CC=CC34)C3C=CCC23)C(CCC(C)(C)C)C1CCC(C)(C)C</smiles>

polyDCPD

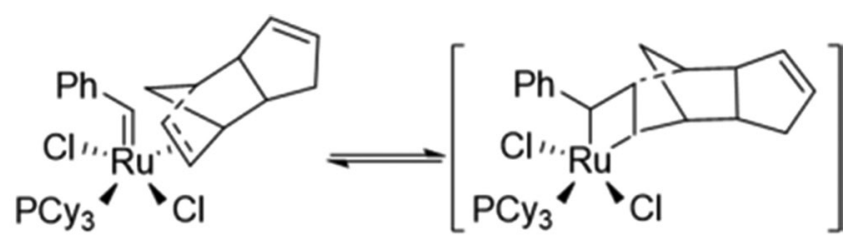<smiles>[3H][CH]</smiles>

Scheme 1: Polymerization route of polyDCPD via ROMP. 

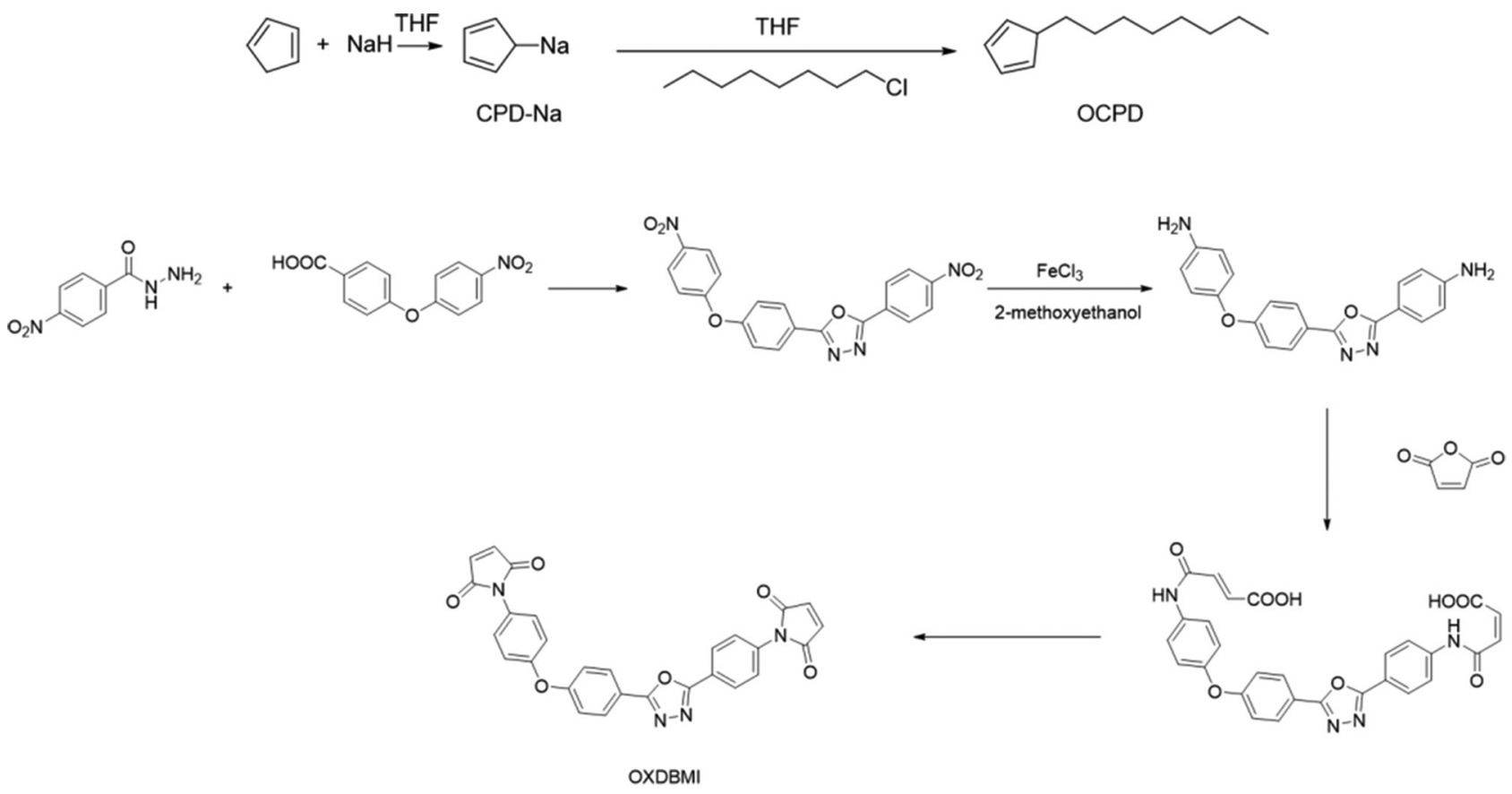

Scheme 2: Synthetic routes of OCPD and OXDBMI monomers.

extra maleic anhydride was removed by precipitation and filtration. Triethylamine and sodium acetate were used to synthesize the final product, OXDBMI. The reaction was heated and refluxed in acetone until the suspension turned into clear, followed by dropwise addition of acetic anhydride and refluxed for another $6 \mathrm{~h}$. The obtained product was precipitated in ice-cold water, collected by filtration and dried under vacuum (Scheme 2) (31).

\subsection{Synthesis of OCPD/polyDCPD, MPBM/ polyDCPD, and OXDBMI/polyDCPD crosslinked copolymers}

MPBM was dissolved in dried DCM under vigorous mechanical stirring, followed by the addition of DCPD and the first-generation Grubbs' catalyst [Ru] (DCPD/[Ru] ratio: $1000 / 1$ ).

The weight ratio of MPBM to DCPD was varied from $5 \%$ to $20 \%$. The mixture was transferred into a mould after the solvent was removed under vacuum and the resulting crosslinked copolymer MPBM/polyDCPD was further cured at the following gradient temperature to complete the polymerization: $120^{\circ} \mathrm{C}$ for $2 \mathrm{~h}, 150^{\circ} \mathrm{C}$ for $2 \mathrm{~h}, 180^{\circ} \mathrm{C}$ for $2 \mathrm{~h}$, and $200^{\circ} \mathrm{C}$ for $2 \mathrm{~h}$. All samples were subjected to characterization immediately after cooling down to minimize the surface oxidation.

OCPD/polyDCPD and OXDBMI/polyDCPD copolymers were synthesized and prepared using similar method (Scheme 3).

\section{Results and discussion}

\subsection{Characterization of polyDCPD, OCPD/ polyDCPD, MPBM/polyDCPD and OXDBMI/polyDCPD crosslinked copolymers}

In order to acquire the modified polyDCPD, a series of monomers with olefin moieties (OCPD, MPBM and OXDBMI) were firstly synthesized (Scheme 2). OCPD, MPBM and OXDBMI were then allowed to copolymerize with polyDCPD through ring-opening metathesis polymerization (ROMP) method followed by the further curing process, ultimately obtaining the polyDCPD, OCPD/ polyDCPD, MPBM/polyDCPD and OXDBMI/polyDCPD crosslinked copolymers (Schemes 1 and 3). Since solubility of all of the copolymers were poor due to the crosslinking, the structures of polyDCPD and the crosslinked copolymers were characterized by the FTIR spectra and the results were shown in Figure 1. Compared with polyDCPD, MPBM/polyDCPD successfully achieved crosslinked structure as evidenced by the FTIR analysis which showing the typical bands of $\mathrm{C}=\mathrm{C}$ and $\mathrm{C}-\mathrm{N}$ stretching vibrations of aromatic rings at $1514 \mathrm{~cm}^{-1}$ and $1049 \mathrm{~cm}^{-1}$, and the typical band of $\mathrm{C}=0$ stretching vibration at $1712 \mathrm{~cm}^{-1}$ (Figure 1a-c). The appearance of $\mathrm{C}=\mathrm{O}$ stretching vibration at $1712 \mathrm{~cm}^{-1}$ and the $\mathrm{C}=\mathrm{N}$ stretching vibration at $1614 \mathrm{~cm}^{-1}$ indicated the successfully conjugation of OXDBMI as shown in 


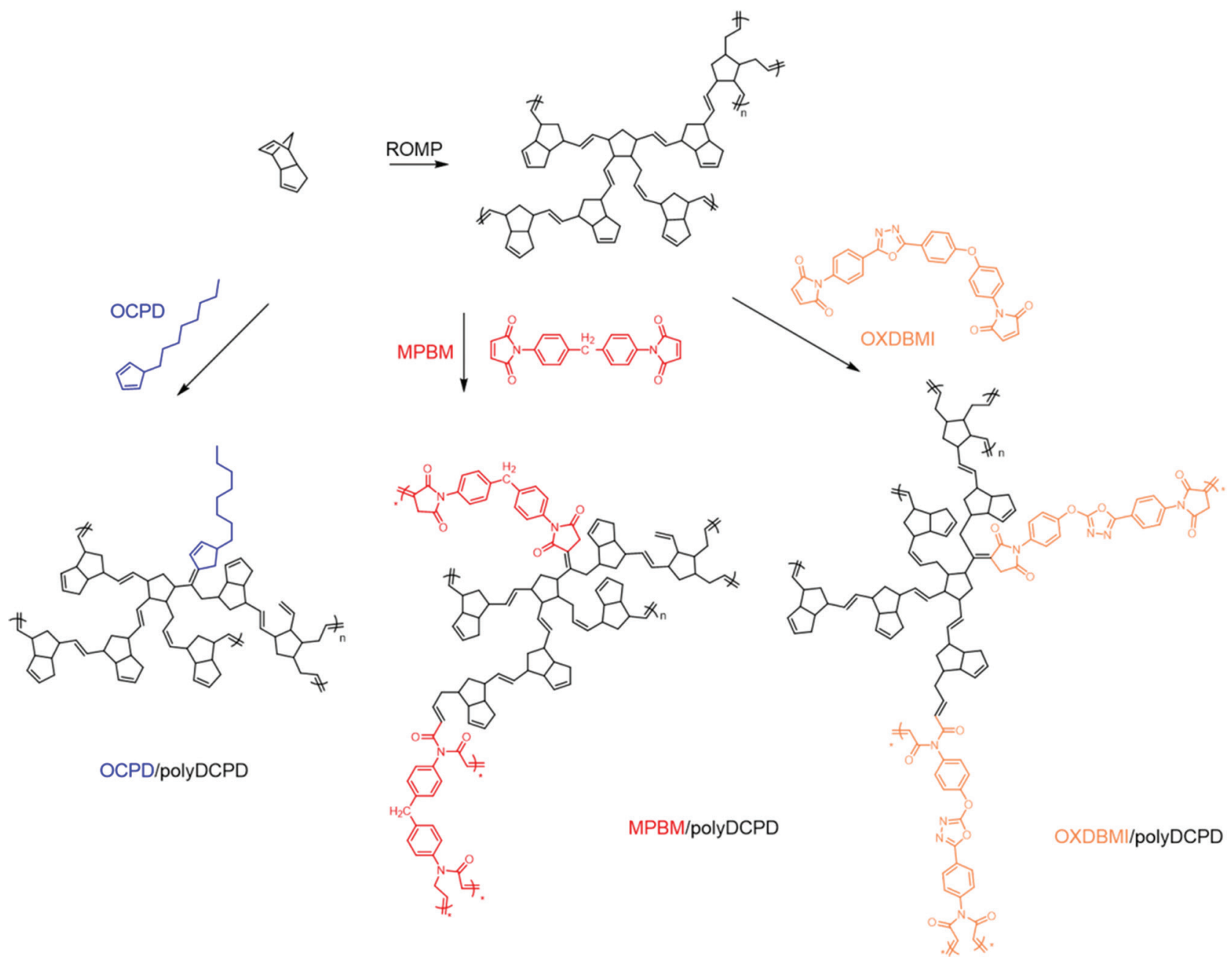

Scheme 3: Polymerization routes of OCPD/polyDCPD, MPBM/polyDCPD and OXDBMI/polyDCPD crosslinked copolymers.

Figure 1d. The appearance of $-\mathrm{CH}_{3}$ band at $1357 \mathrm{~cm}^{-1}$ and $1459 \mathrm{~cm}^{-1}$ indicated the successfully conjugation of OCPD as shown in Figure 1e.

\subsection{Thermal stability of polyDCPD, OCPD/ polyDCPD, MPBM/polyDCPD and OXDBMI/polyDCPD crosslinked copolymers}

To investigate the thermal stability of the second monomer modified polyDCPD and optimize the obtained materials, TGA was performed to characterize the decomposition temperatures at 5\%,10\%, 20\% and 50\% weight loss (Td, 5\%, Td, 10\%, Td, 20\% and Td, 50\%), and the residual weight at $800^{\circ} \mathrm{C}(\mathrm{RW})$, respectively. The results of each material are summarized in Table 1. As shown in Table 1, the incorporation of all of the selected monomers exhibited improved thermal stability as indicated by the improved decomposition temperature at various weight losses and the residual weight at $800^{\circ} \mathrm{C}$. Upon optimization, the incorporation of aliphatic and flexible monomer, OCPD, displayed a least enhanced decomposition temperature at the weight loss from $20 \%$ to $50 \%$, as well as a slight increase RW value. However, the incorporation of the aromatic and rigid monomer, MPBM, displayed a significant increase of the decomposition temperature at the full temperature range. These results were mainly due to the excellent thermal stability of the aromatic materials and could also be attributed to the inhibition of the potential back-biting process of polyDCPD (28). Interestingly, the final RW value was not as high as expected, which mainly owed to the incompatibility of the flexible DCPD monomer and the rigid MPBM monomer (13). As a result, the combination of both flexible and rigid property into the inserted monomer should be the optimized strategy to improve the thermal stability and maintain the desired RW. As shown in Table 1 
a

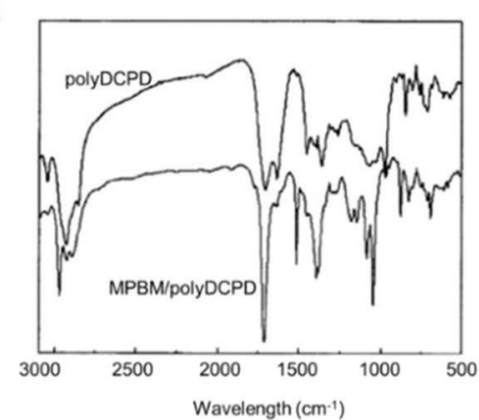

b

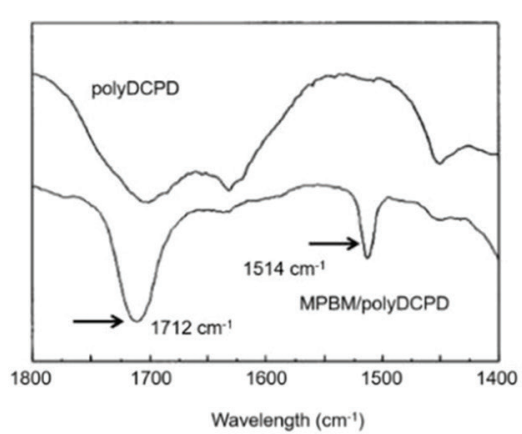

C

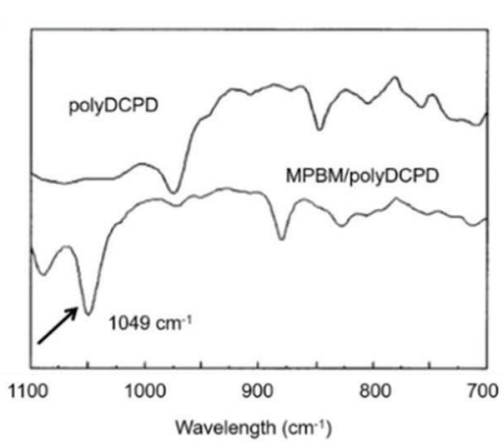

e

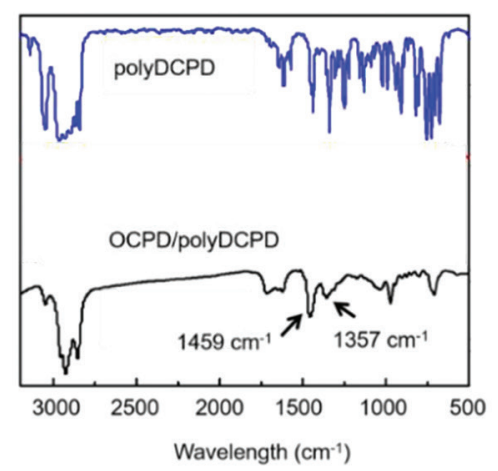

Figure 1: FTIR spectra of MPBM/polyDCPD at various wavelength ranges (a: full range from $3000^{-1}$ to $500 \mathrm{~cm}^{-1}, \mathrm{~b}$ : from $1800^{-1}$ to $1400 \mathrm{~cm}^{-1}, \mathrm{c}$ : from $1100^{-1}$ to $\left.700 \mathrm{~cm}^{-1}\right)$, OXDBMI/polyDCPD with various OXDBMI weight ratios (5\%, $10 \%$ and $20 \%$ ) (d) and OCPD/polyDCPD (e) crosslinked copolymers.

Table 1: Parameters obtained from TGA curves of polyDCPD and copolymers.

\begin{tabular}{|c|c|c|c|c|c|}
\hline Sample name & $T d, 5 \%\left({ }^{\circ} \mathrm{C}\right)^{\mathrm{a}}$ & $T d, 10 \%\left({ }^{\circ} \mathrm{C}\right)^{\mathrm{a}}$ & $T d, 20 \%\left({ }^{\circ} \mathrm{C}\right)^{\mathrm{a}}$ & $T d, 50 \%\left({ }^{\circ} \mathrm{C}\right)^{\mathrm{a}}$ & $\mathrm{RW}(\%)^{\mathrm{b}}$ \\
\hline polyDCPD & 383.7 & 452.4 & 471.2 & 476.3 & 16.3 \\
\hline OCPD/polyDCPD (5\%) & 384.5 & 454.2 & 471.6 & 477.9 & 20.8 \\
\hline OCPD/polyDCPD (10\%) & 385.4 & 454.9 & 472.1 & 480.2 & 20.9 \\
\hline OCPD/polyDCPD (20\%) & 388.9 & 457.2 & 473.9 & 480.3 & 21.1 \\
\hline MPBM/polyDCPD (5\%) & 389.2 & 451.3 & 487.9 & 503.5 & 22.3 \\
\hline MPBM/polyDCPD (10\%) & 390.5 & 454.9 & 488.6 & 510.2 & 26.2 \\
\hline MPBM/polyDCPD (20\%) & 392.1 & 457.8 & 491.8 & 513.7 & 28.3 \\
\hline OXDBMI/polyDCPD (5\%) & 335.4 & 433.8 & 474.4 & 474.9 & 20.2 \\
\hline OXDBMI/polyDCPD (10\%) & 319.4 & 434.2 & 475.2 & 475.4 & 25.3 \\
\hline OXDBMI/polyDCPD (20\%) & 403.5 & 446.1 & 479.9 & 487.2 & 34.8 \\
\hline
\end{tabular}

${ }^{a}$ Temperature at $5 \%, 10 \%, 20 \%, 50 \%$ weight loss obtained from TGA results, respectively. ${ }^{\mathrm{b}}$ Residual weight percentage at $800^{\circ} \mathrm{C}$ from TGA.

and Figure 2, even the final decomposition temperature of the OXDBMI/polyDCPD crosslinked copolymers was not as high as that of MPBM/polyDCPD crosslinked copolymers, the final RW value increased from $16.3 \%$ to $34.8 \%$. Furthermore, the incorporation weight ratio of the OXDBMI was varied to evaluate the thermal stability of the crosslinked copolymers and the results indicated a positive correlation conclusion. The major weight loss happened at the temperature from $450^{\circ} \mathrm{C}$ to $500^{\circ} \mathrm{C}$, which corresponded to the degradation of the aliphatic structures of the cured polyDCPD network. Due to the aromatic property of OXDBMI monomer, it was obvious that the thermal stability enhanced with the increased ratio of OXDBMI in the copolymers. 


\subsection{Dynamic mechanical properties of polyDCPD, OCPD/polyDCPD, MPBM/ polyDCPD and OXDBMI/polyDCPD crosslinked copolymers}

The dynamic mechanical property of polyDCPD and crosslinked copolymers was evaluated by DMA. The viscoelasticity of the materials was shown in Figure 3 and the storage modulus (at both glass region and rubber region) and the glass transition temperatures were summarized in Table 2. As shown in Table 2, the storage modulus G' of the copolymers in the glass state (storage modulus at $303 \mathrm{~K}$ ) increased with the incorporation of either OCPD, MPBM or OXDBMI, which mainly due to the crosslinked network structures of the cured copolymers.

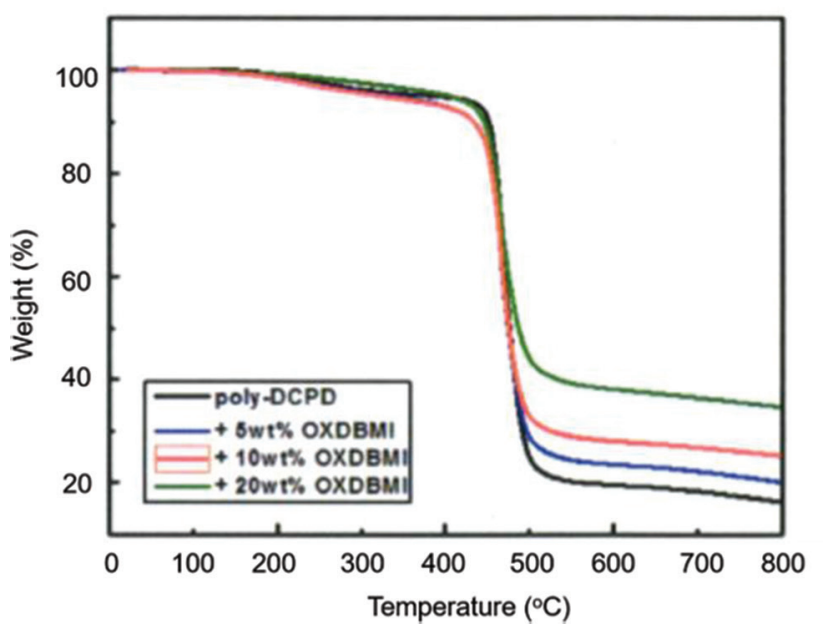

Figure 2: TGA curves of OXDBMI/polyDCPD with various OXDBMI incorporation weight ratios (5\%, $10 \%$ and $20 \%)$.

a

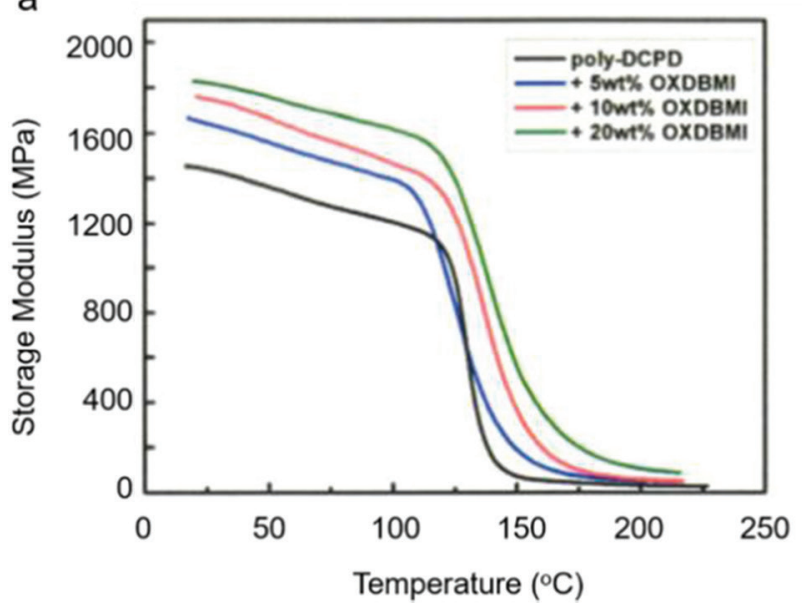

OCPD monomer showed the slightest increase because of the flexible property of the aliphatic chain. While the MPBM owning the rigid and stiff aromatic rings in the structure was expected to exhibit significantly enhanced storage modulus, G' at both $303 \mathrm{~K}$ (temperature below $\mathrm{Tg}$ ) and $\mathrm{Tg}+50 \mathrm{~K}$ (temperature above $\mathrm{Tg}$ ) were not as high as those of OXDBMI. This result was mainly because of the interaction effect between the polyDCPD and the second monomer. As is well known that the aromatic rings adopted extremely stiffness, the compatibility and the interaction between the MPBM and the polyDCPD was dramatically poor compared with OXDBMI. Interestingly, the increase rate of $\mathrm{Tg}$ slowed down with the incorporation ratio of OXDBMI higher than $10 \%$, which could be explained by the reduced crosslinking density when extra monomers inserted.

\subsection{Mechanical property of OXDBMI/ polyDCPD crosslinked copolymers}

To evaluate the mechanical properties of the most optimized monomer, OXDBMI, modified polyDCPD, flexural strength/modulus and impact strength tests were performed. As shown in Figures $4 \mathrm{a}$ and 4b, all the OXDBMI modified polyDCPD showed notably higher flexural strength and flexural modulus, as well as the impact strength, than the unmodified polyDCPD by up to 2-folds, which indicated that the incorporation of optimized OXDBMI monomer greatly enhanced their mechanical properties. Remarkably, both the flexural strength and the impact strength enhanced with the increase of OXDBMI, peaking at the OXDBMI weight ratio of $10 \mathrm{wt} \%$, indicating that the rigidity and the

b

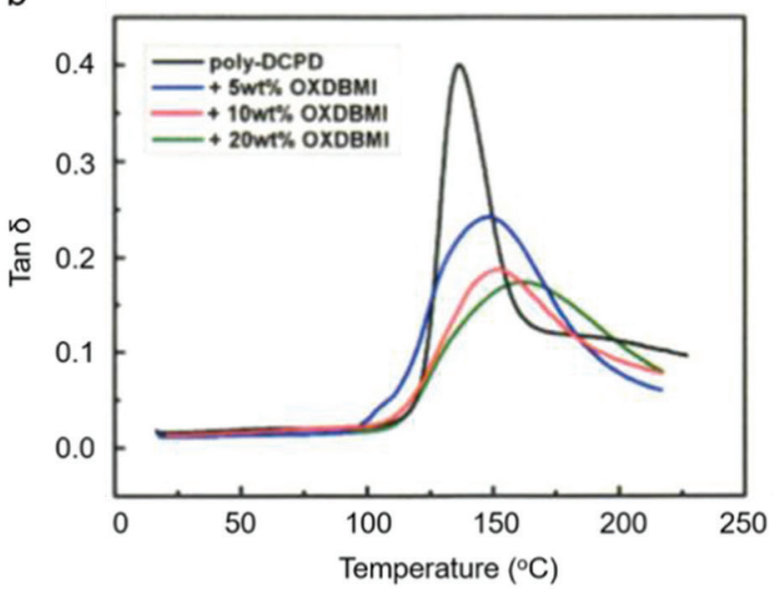

Figure 3: Dynamic mechanical properties of OXDBMI/polyDCPD crosslinked copolymers based on DMA measurements. (a) Storage modulus of OXDBMI/polyDCPD. (b) Tan $\delta$ of OXDBMI/polyDCPD. 
crosslinking effect of the OXDBMI were essential to be optimized. However, an increase in the OXDBMI weight ratio from $10 \mathrm{wt} \%$ to $20 \mathrm{wt} \%$ led to a significant

Table 2: Parameters obtained from the DMA thermograms for polyDCPD and copolymers.

\begin{tabular}{lcrr}
\hline Sample name & $\begin{array}{c}\mathbf{G}^{\prime}{ }^{303 \mathrm{~K}} \\
(\mathbf{M P a})^{\mathrm{a}}\end{array}$ & $\begin{array}{r}\mathbf{G}^{\prime}{ }_{\mathrm{T}+50 \mathrm{~K}} \\
(\mathbf{M P a})^{\mathbf{b}}\end{array}$ & $\mathbf{T}_{\mathbf{g}} \mathbf{M P a}^{\mathrm{c}}$ \\
\hline polyDCPD & 1426.1 & 37.1 & 136.0 \\
OCPD/polyDCPD (5\%) & 1481.3 & 42.5 & 140.2 \\
OCPD/polyDCPD (10\%) & 1500.1 & 49.8 & 144.7 \\
OCPD/polyDCPD (20\%) & 1512.8 & 50.3 & 145.9 \\
MPBM/polyDCPD (5\%) & 1521.3 & 59.4 & 150.3 \\
MPBM/polyDCPD (10\%) & 1799.6 & 60.3 & 159.8 \\
MPBM/polyDCPD (20\%) & 1802.5 & 87.5 & 153.2 \\
OXDBMI/polyDCPD (5\%) & 1628.6 & 53.5 & 148.4 \\
OXDBMI/polyDCPD (10\%) & 1738.4 & 56.6 & 152.4 \\
OXDBMI/polyDCPD (20\%) & 1811.2 & 90.8 & 161.8 \\
\hline
\end{tabular}

a Storage modulus (G') at $303 \mathrm{~K}$. ${ }^{\mathrm{b}}$ Storage modulus (G') at $\mathrm{T}_{\mathrm{g}}+50 \mathrm{~K}$.

${ }^{\mathrm{C}}$ Defined as the temperature of maximum tan $\delta$. decrease in both flexural strength/modulus and impact strength, which could be attributed to the excessive inserted monomers inhibiting the crosslinking process by hampering the interactions between polyDCPD and OXDBMI. Moreover, extra OXDBMI would lead to the irreversible local defects in the materials, as shown in the SEM images in Figure 4c, which finally limited the improvement of the impact strength as well as other mechanical properties.

\section{Conclusion}

In conclusion, we have developed a copolymer system which consisted of dicyclopentadiene and a library of designed monomers with different stiffnesses and rigidities which can reinforce original polydicyclopentadiene. In addition, we demonstrated that the incorporation of the second monomer with aromatic and bismaleimide moieties significantly altered their crosslinking to promote the thermal stability and mechanical properties. More importantly, we unraveled a

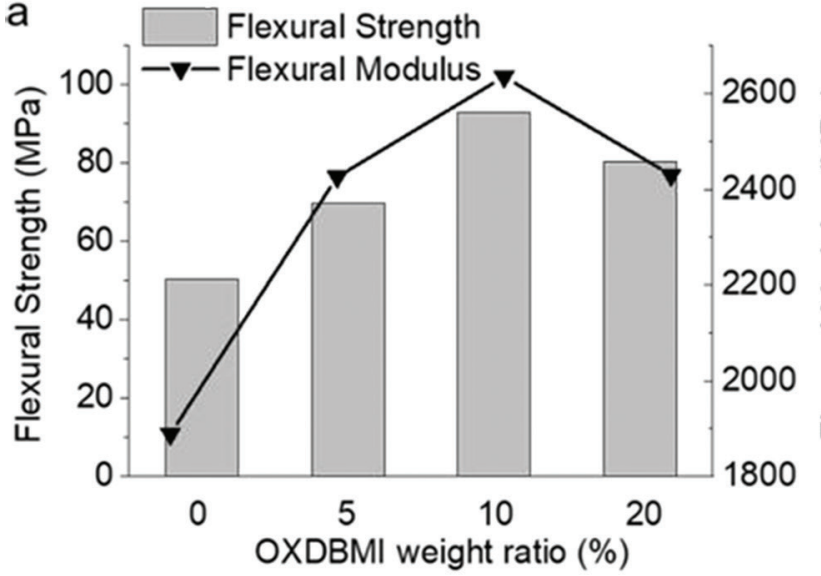

b

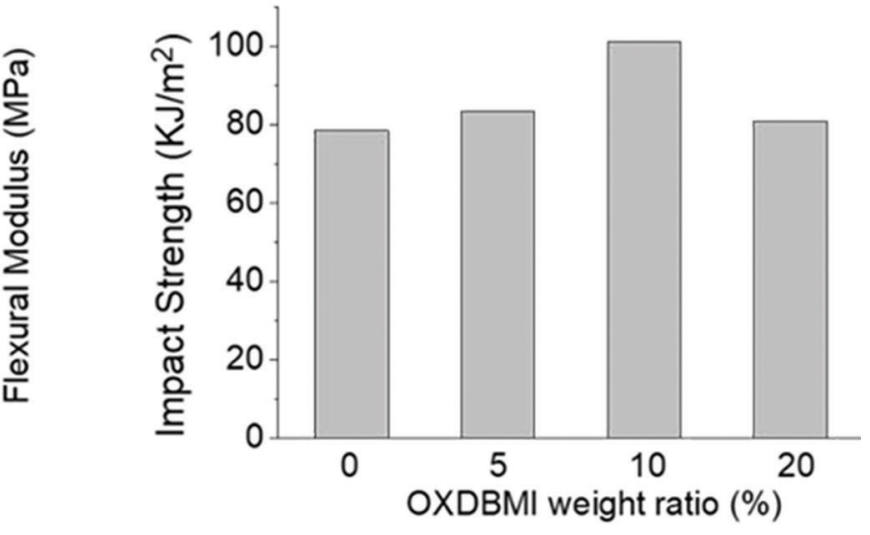

C
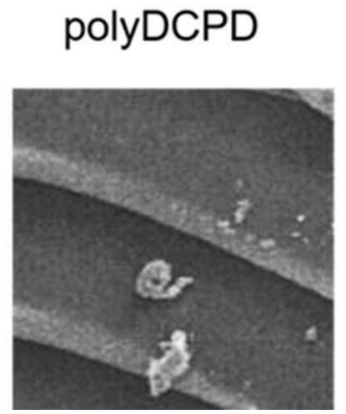

OXDBMI/polyDCPD (5 wt\%)

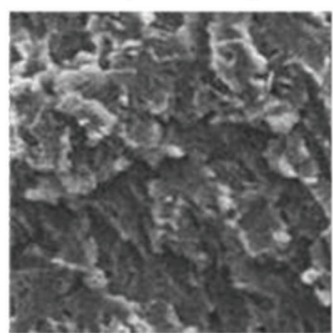

\section{OXDBMI/polyDCPD OXDBMI/polyDCPD}

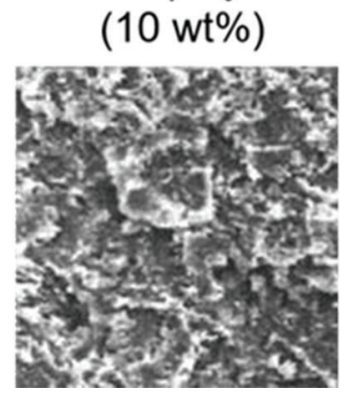

(20 wt\%)

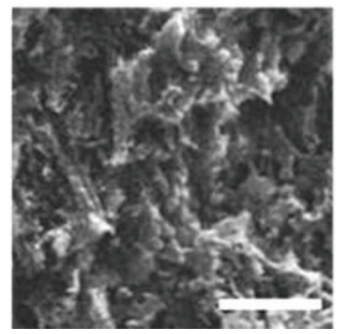

Figure 4: Mechanical properties of OXDBMI/polyDCPD crosslinked copolymers evaluated using flexural strength/modulus, impact strength and SEM images. (a) Flexural strength/modulus of OXDBMI/polyDCPD. (b) Impact strength of OXDBMI/polyDCPD. (c) SEM images of the fractured surfaces of OXDBMI/polyDCPD crosslinked copolymers. Bar $=50 \mu \mathrm{m}$. 
that the rigidity and stiffness could be optimized due to the compatibility with the polyDCPD. Consequently, the combination of both flexible chain and rigid part into one bismaleimide-like monomer was the optimal strategy to design the incorporated monomer for the polyDCPD reinforcing. The aromatic motifs could enhance the thermal stability of the material and the flexible chains could promote the interaction with the DCPD monomers. Such understanding and approach described here provides a promising structural design strategy which could be applied to manipulate the thermal and dynamic mechanical properties of polyDCPD efficiently and successfully.

Acknowledgments: Y. Z. acknowledges the support from the National Natural Science Foundation of China (51703018) and the Fundamental Research Funds for the Central Universities (No. DUT16RC(3)114).

Conflict of interest statement: Y.Z. declares no potential conflicts of interest with respect to the research, authorship, and/or publication of this article.

\section{References}

1. Buchmeiser M.R., Homogeneous metathesis polymerization by well-defined group VI and group VIII transition-metal alkylidenes: fundamentals and applications in the preparation of advanced materials. Chem. Rev., 2000, 100, 1565-1604.

2. Buck M.E., Lynn D.M., Azlactone-functionalized polymers as reactive platforms for the design of advanced materials: progress in the last ten years. Polym. Chem., 2012, 3, 66-80.

3. Monsaert S., Vila A.L., Drozdzak R., Van Der Voort P., Verpoort F., Latent olefin metathesis catalysts. Chem. Soc. Rev., 2009, 38, 3360-3372.

4. Ban H.T., Shigeta M., Nagamune T., Uejima M., Synthesis of cyclo-olefin copolymer latexes and their carbon nanotube composite nanoparticles. J. Polym. Sci. Pol. Chem., 2013, 51, 4584-4591.

5. Bellan L.M., Coates G.W., Craighead H.G., Poly (dicyclopentadiene) submicron fibers produced by electrospinning. Macromol. Rapid. Commun., 2006, 27, 511-515.

6. Perring M., Bowden N.B., Assembly of organic monolayers on polydicyclopentadiene. Langmuir, 2008, 24, 10480-10487.

7. Ruiu A., Sanna D., Alzari V., Nuvoli D., Mariani A., Advances in the frontal ring opening metathesis polymerization of dicyclopentadiene. J. Polym. Sci. Pol. Chem., 2014, 52, 2776-2780.

8. Robertson I.D., Pruitt E.L., Moore J.S., Frontal ring-opening metathesis polymerization of exo-dicyclopentadiene for low catalyst loadings. ACS Macro. Lett., 2016, 5, 593-596.

9. Cuthbert T.J., Li T., Speed A.W.H., Wulff J.E., Structure of the thermally induced cross-link in C-linked methyl ester-functionalized polydicyclopentadiene (fPDCPD). Macromolecules, 2018, 51, 2038-2047.
10. Della Martina A., Hilborn J.G., Muhlebach A., Macroporous cross-linked poly(dicyclopentadiene). Macromolecules, 2000, 33, 2916-2921.

11. Yoonessi M., Toghiani H., Daulton T.L., Lin J.S., Pittman C.U., Clay delamination in clay/poly(dicyclopentadiene) nanocomposites quantified by small angle neutron scattering and high-resolution transmission electron microscopy. Macromolecules, 2005, 38, 818-831.

12. Jeong W., Kessler M.R., Toughness enhancement in ROMP functionalized carbon nanotube/polydicyclopentadiene composites. Chem. Mat., 2008, 20, 7060-7068.

13. Hu F., Zheng Y., Fang Y., Ren X., Liu X., Preparation and properties of high performance phthalide- containing bismaleimide reinforced polydicyclopentadiene. J. Appl. Polym. Sci., 2014, 131, 40474 (1-6).

14. Novoselov K.S., Geim A.K., Morozov S.V., Jiang D., Zhang Y., Dubonos S.V., et al., Electric field effect in atomically thin carbon films. Science, 2004, 306, 666-669.

15. Simons R., Guntari S.N., Goh T.K., Qiao G.G., Bateman S.A., Poly(dicyclopentadiene)-montmorillonite nanocomposite formation via simultaneous intergallery-surface initiation and chain crosslinking using ROMP. J. Polym. Sci. Pol. Chem., 2012, 50, 89-97.

16. Maity J., Jacob C., Das C.K., Kharitonov A.P., Singh R.P., Alam S., Fluorinated aramid fiber reinforced polypropylene composites and their characterization. Polym. Compos., 2007, 28, 462-469.

17. Yoonessi M., Toghiani H., Kingery W.L., Pittman C.U., Preparation, characterization, and properties of exfoliated/delaminated organically modified clay/dicyclopentadiene resin nanocomposites. Macromolecules, 2004, 37, 2511-2518.

18. Kim J.H., Kim Y.H., Kim Y.J., Won J.C., Choi K.Y., Synthesis and characterization of novel aromatic condensation polymers containing rigid benzoxazole pendent groups. J. Appl. Polym. Sci., 2004, 92, 178-185.

19. Chen C.C., Wang L.F., Wang J.J., Hsu T.C., Chen C.F., Diacid architecture effect on the synthesis and microstructure of rigid-rod poly(benzobisimidazoles). J. Mater. Sci., 2002, 37, 4109-4115.

20. Sandhya K.Y., Pillai C.K.S., Sato M., Tsutsumi N., Highly stable rigid main-chain nonlinear optical polymers with nematic phase: effect of liquid-crystalline phase on nonlinear optical response. J. Polym. Sci. Pol. Chem., 2003, 41, 1527-1535.

21. Rao K.V., Haldar R., Maji T.K., George S.J., Porous polyimides from polycyclic aromatic linkers: selective $\mathrm{CO}_{2}$ capture and hydrogen storage. Polymer, 2014, 55, 1452-1458.

22. Dotrong M., Dotrong M.H., Evers R.C., Multidimensional benzobisoxazole rigid-rod polymers .I. preparation and characterization. J. Polym. Sci. Pol. Chem., 1997, 35, 3451-3456.

23. Devaux E., Caza C., Recher G., Bielarski D., Characterization of interfacial adhesion in a ultra-high-molecular-weight polyethylene reinforced polydicyclopentadiene composite. Polym. Test., 2002, 21, 457-462.

24. Mahesh K.P.O., Alagar M., Preparation and characterization of chain-extended bismaleimide modified polyurethane-epoxy matrices. J. Appl. Polym. Sci., 2003, 87, 1562-1568.

25. Vanaja A., Rao R.M.V., Synthesis and characterisation of epoxynovolac/bismaleimide networks. Eur. Polym. J., 2002, 38, 187-193.

26. Dinakaran K., Alagar M., Kumar R.S., Preparation and characterization of bismaleimide/1,3-dicyanatobenzene modified 
epoxy intercrosslinked matrices. Eur. Polym. J., 2003, 39, 2225-2233.

27. Xiong X., Chen P., Zhang J., Yu Q., Preparation and properties of high performance phthalide-containing bismaleimide modified epoxy matrices. J. Appl. Polym. Sci., 2011, 121, 3122-3130.

28. Hu F., Zheng Y., Fang Y., Preparation and properties of cyclopentadiene-containing monomer modified polydicyclopentadiene. Polymer, 2014, 55, 2809-2816

29. Delancey J.M., Cavazza M.D., Rendos M.G., Ulisse C.J., Palumbo S.G., Mathers R.T., Controlling crosslinking in thermosets via chain transfer with monoterpenes. J. Polym. Sci. Pol. Chem., 2011, 49, 3719-3727.

30. Liu Z., Chen P., Zhang X.L., Yu Q., Degradation of plasma-treated poly(p-phenylene benzobisoxazole) fiber and its adhesion with bismaleimide resin. RSC Adv., 2014, 4, 3893-3899.

31. Kelsey D.R., Chuah H.H., Ellison R.H., Handlin D.L., Scardino B.M., Ring-opened metathesis polymers from cyclohexenylnorbornene and related Diels-Alder adducts of cyclopentadiene with vinyl and divinyl cyclohydrocarbons. J. Polym. Sci. Pol. Chem., 1997, 35, 3049-3063. 